This item was submitted to Loughborough's Research Repository by the author.

Items in Figshare are protected by copyright, with all rights reserved, unless otherwise indicated.

\title{
Modelling of High Redundancy Actuation utilising multiple moving coil actuators
}

PLEASE CITE THE PUBLISHED VERSION

PUBLISHER

(C) The International Federation of Automatic Control (IFAC)

LICENCE

CC BY-NC-ND 4.0

REPOSITORY RECORD

Davies, Jessica, Thomas Steffen, Roger Dixon, Roger M. Goodall, Argyrios C. Zolotas, and John T. Pearson. 2019. "Modelling of High Redundancy Actuation Utilising Multiple Moving Coil Actuators". figshare.

https://hdl.handle.net/2134/3672. 
This item was submitted to Loughborough's Institutional Repository by the author and is made available under the following Creative Commons Licence conditions.

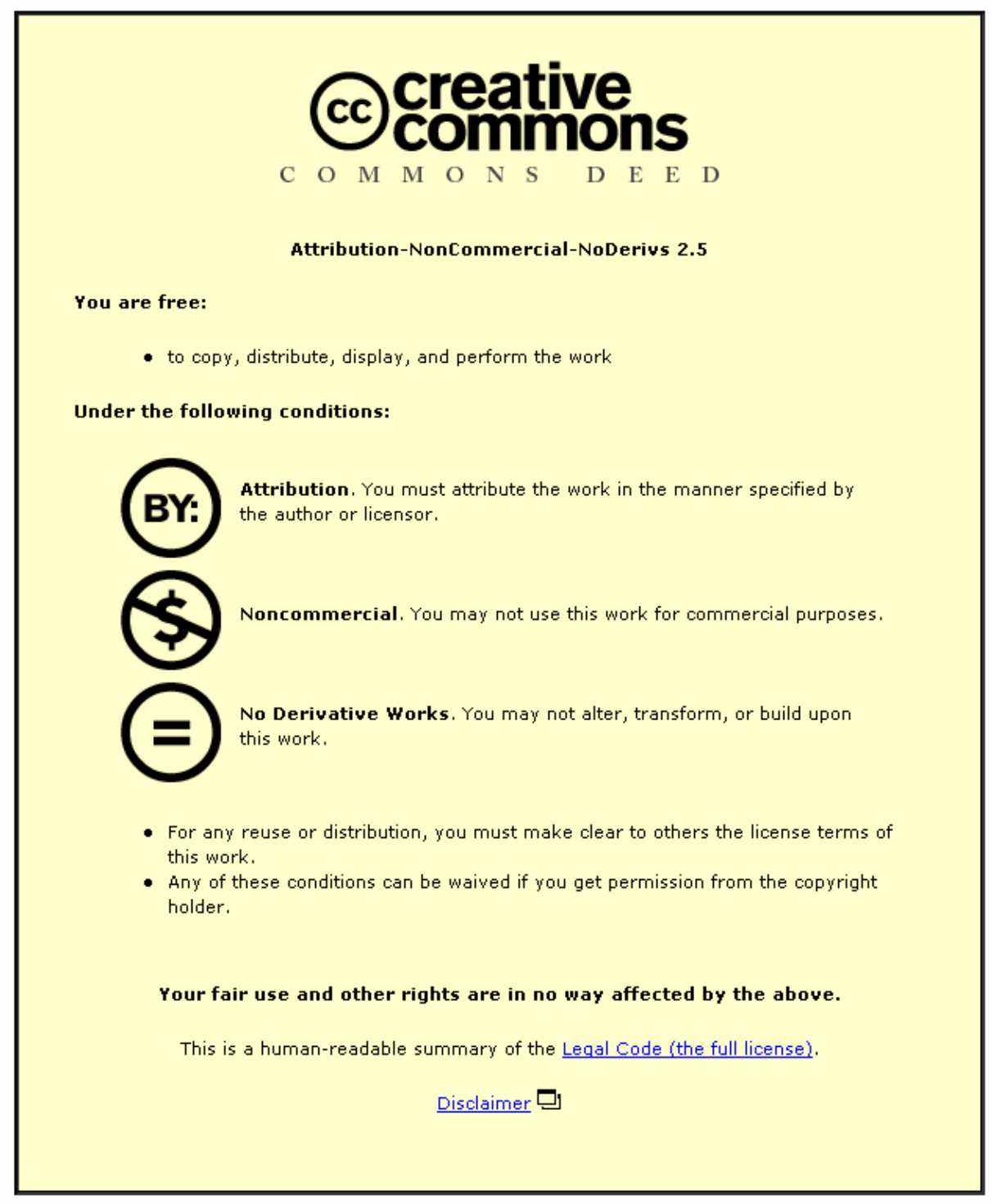

For the full text of this licence, please go to: http://creativecommons.org/licenses/by-nc-nd/2.5/ 


\title{
Modelling of High Redundancy Actuation Utilising Multiple Moving Coil Actuators
}

\author{
J. Davies * T. Steffen ${ }^{*}$ R. Dixon ${ }^{*}$ R.M. Goodall * \\ A.C. Zolotas * J. Pearson ${ }^{* *}$ \\ * Control Systems Group, Loughborough University, Loughborough, \\ LE11 3TU, UK, http://www.lboro.ac.uk/departments/el/research/scg \\ ** SEIC, BAE Systems, Holywell Park, Loughborough, LE11 3TU, UK, \\ http://www.seic-loughborough.com
}

\begin{abstract}
This paper presents the modelling of a moving coil actuator for use as an element in a High Redundancy Actuator (HRA). A single element model is derived from first principles and verified using experimental data. This model is subsequently used to describe an approach to deriving models of multi-element HRAs and determine the effect of a variety of faults, chosen to be appropriate for the electro-magnetic technology, on the behaviour of multi-element assemblies.
\end{abstract}

\section{INTRODUCTION}

\subsection{Fault Tolerant Control and Actuator Redundancy}

A fault may be defined as a defect or imperfection that occurs in the hardware or software of a system. Faults in automated processes will often cause undesired reactions which could manifest as failures, where an expected action is not completed by the overall system. The consequences of failures could include damage to the plant, its environment, or people in the vicinity of that plant [Blanke et al., 2001]. Fault tolerant control aims to prevent failures and achieve adequate system performance in the presence of faults.

The majority of research to date has concentrated on sensor faults. Significant advances have been made in this area, however, most of these strategies are not applicable to actuator faults. This is attributable to the fundamental differences between actuators and sensors. Sensors deal with information, and measurements may be processed or replicated analytically to provide fault tolerance. However, actuators must deal with energy conversion, and as a result actuator redundancy is essential if fault tolerance is to be achieved in the presence of actuator faults. Actuation force will always be required to keep the system in control and bring it to the desired state [Patton, 1991]. No approach can avoid this fundamental requirement.

The common solution is to use some form of over-actuation in which the fault-free system has more control action than needed. For critical systems, the normal approach involves straightforward replication of the actuators, e.g. 3 or 4 actuators are used in parallel for aircraft flight control systems. Each redundant actuator must be capable of performing the task alone and possibly override the other faulty actuators. This over-engineering however, incurs penalties as cost and weight are increased and subsequently efficiency is reduced.

\subsection{High Redundancy Actuation}

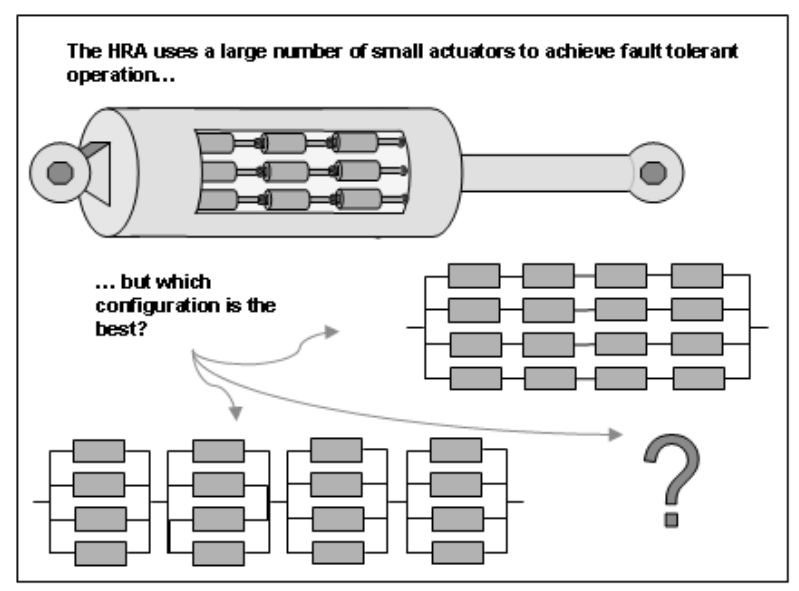

Figure 1. High Redundancy Actuator.

The High Redundancy Actuator (HRA) concept is a novel approach to actuator fault tolerance, inspired by human musculature. A muscle is composed of many individual muscle cells, each of which provides a minute contribution to the force and the travel of the muscle. These properties allow the muscle, as a whole, to be highly resilient to individual cell damage.

The HRA project aims to use the same principle of cooperation to provide intrinsic fault tolerance using existing technology. To achieve this, a high number of small actuator elements are assembled in parallel and in series to form one highly redundant actuator (see Figure 1). Faults within the actuator will affect the maximum capability, but through robust control, full performance can be maintained without either adaptation or reconfiguration.

The HRA is an important new approach within the overall area of fault-tolerant control. When applicable, it can provide actuators that gracefully degrade, and that continue 
to operate at close to nominal performance in the presence of multiple faults in the actuator elements. The HRA research project has already studied the use of electromechanical technology [Du et al., 2007] in order to assess the concept's viability. Progress towards an electromagnetic HRA is also under way [Steffen et al., 2007a].

\subsection{Overview}

This paper presents the modelling of a moving coil actuator that is intended to be the building block of an electro-magnetic HRA. Derivation of the element model from operating principles and equivalent circuits is provided in Section 2. Section 3 describes the experimental identification of the parameters and verification of the model. The modelling of faults in a single element is given in Section 4. Section 5 details the modelling of element assemblies, the effect of faults in which is discussed in Section 6. Finally, the paper's conclusions are made in Section 7 which includes comments on the future direction of this research.

\section{MODELLING OF A SINGLE ACTUATION ELEMENT}

In order to construct a multi-element actuation system, it is first necessary to model a single actuation element i.e. a moving coil actuator provided by SMAC UK Ltd. [SMAC, 2004]. This modelling will be addressed here.

\subsection{Operating Principles}

Figure 2 illustrates the basic components a moving coil

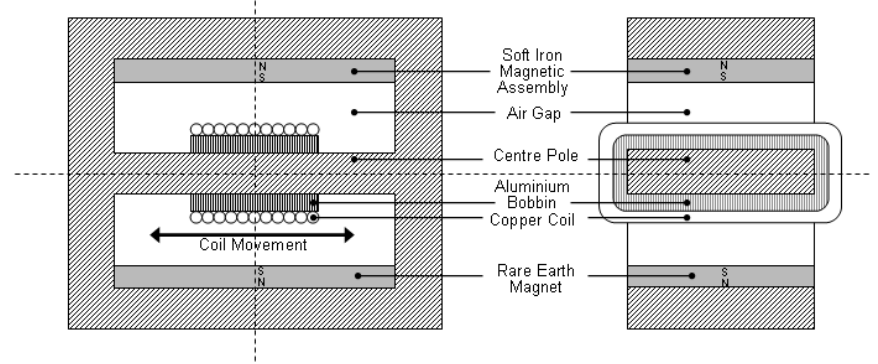

Figure 2. Moving Coil Actuator.

actuator. It comprises a moving coil wound round the centre pole of a magnetic assembly that produces a uniform magnetic field perpendicular to the current conducted in the coil. On providing a voltage, a current flows in the coil generating a force which is parallel to the direction of travel. This force causes the coil, and the rod which is mounted to it, to move. The force is proportional to the current in the coil, the number of turns, and the flux strength.

The copper coil is wound round an aluminium bobbin, which forms part of the piston carriage. This aluminium bobbin surrounds the centre pole of the magnet, forming a circuit, and as such, as it moves within the magnetic field, eddy currents are induced within it. These eddy currents produce magnetic fields that oppose the external magnetic field and thus oppose the movement of the coil causing a

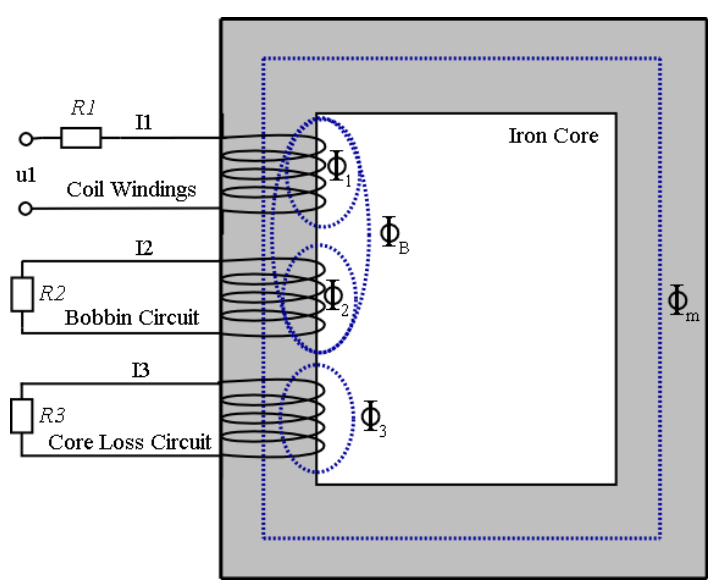

Figure 3. Magnetic flux within the static system.

damping effect. In addition, eddy currents are also induced within the bobbin by the changing current in the coil. This aspect of the moving coil actuator in question complicates the modelling procedure as the usual resistor inductor circuit that is used to model the electrical characteristics is no longer appropriate. Hence, a different approach, based on magnetic principles is taken to the modelling of this system.

As the system contains electrical, magnetic and mechanical elements, electrical analogies will be used to derive one homogeneous model. The actuation element will be modelled in two stages: firstly the electrical subsystem which characterises the force produced by the electrical input, and then the mechanical subsystem upon which this force is acting. Equivalent circuits will be formulated for both subsystems and then they will be combined using dynamical laws to produce one overall equivalent circuit for the element.

\subsection{Electrical Subsystem}

Figure 3 illustrates the flux within the system. The figure shows the iron core surrounded by three coil circuits: the moving coil with its voltage input $u_{1}$ and winding resistance $R_{1}$; a second circuit representing the bobbin, which is effectively a closed-turn with resistance $R_{2}$; and a third coil representing the inductive and resistive core losses. The majority of the flux flows in the iron core, and is shown in Figure 3 as $\Phi_{M} . \Phi_{1}$ is the flux linking the coil and $\Phi_{2}$ is the flux flowing in the bobbin. $\Phi_{b}$ is the flux that links the coil and the bobbin. Finally, the core losses are denoted as $\Phi_{3}$.

Using the following expressions for the magnetomotive force (m.m.f.) that creates the flux and the electromotive force (e.m.f.) created across the coils by the changing flux:

$$
\begin{aligned}
& \mathcal{F}=\Re \Phi \\
& \mathrm{E}=N \frac{d \Phi}{d t}
\end{aligned}
$$

The three circuit equations can be defined:

$$
\begin{aligned}
\mathrm{u}_{1} & =N_{1} \frac{d}{d t}\left(\Phi_{M}+\Phi_{1}+\Phi_{B}\right)+R_{1} \mathrm{I}_{1} \\
0 & =N_{2} \frac{d}{d t}\left(\Phi_{M}+\Phi_{2}+\Phi_{B}\right)+R_{2} \mathrm{I}_{2}
\end{aligned}
$$




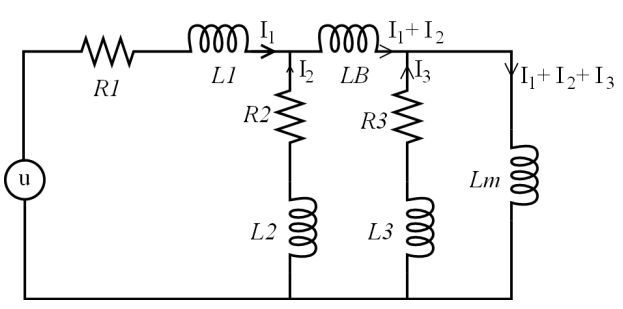

Figure 4. Electrical subsystem equivalent circuit.

$$
0=N_{3} \frac{d}{d t}\left(\Phi_{M}+\Phi_{3}\right)+R_{3} \mathrm{I}_{3}
$$

and using the m.m.f. law, analogous to AMPERE's law, $\Re \Phi=N I$ and substituting terms in $\frac{N}{\Re}$ for inductances gives:

$$
\begin{aligned}
\mathrm{u}_{1} & =N_{1}\left(L_{M} \frac{d \mathrm{I}_{m}}{d t}+L_{1} \frac{d \mathrm{I}_{1}}{d t}+L_{B} \frac{d}{d t}\left(\mathrm{I}_{1}+\mathrm{I}_{2}\right)\right)+R_{1} \mathrm{I}_{1} \\
0 & =N_{2}\left(L_{M} \frac{d \mathrm{I}_{m}}{d t}+L_{2} \frac{d \mathrm{I}_{2}}{d t}+L_{B} \frac{d}{d t}\left(\mathrm{I}_{1}+\mathrm{I}_{2}\right)\right)+R_{2} \mathrm{I}_{2} \\
0 & =N_{3}\left(L_{M} \frac{d \mathrm{I}_{m}}{d t}+L_{3} \frac{d \mathrm{I}_{3}}{d t}\right)+R_{3} \mathrm{I}_{3}
\end{aligned}
$$

where $\mathrm{I}_{m}=\mathrm{I}_{1}+\mathrm{I}_{2}+\mathrm{I}_{3}$. These equations describe the element without mechanical movement i.e. when the bobbin is clamped. Hence, the mode represents only the electrical subsystem. Some simplifications may be made as $L_{2}$ and $L_{3}$ are much smaller than $L_{m}$ and $L_{B}$ and thus they may be removed with little affect on the system [Chai, 1998]. The resultant equivalent circuit is shown in Figure 4 , and the following transfer function may be derived:

$$
\frac{\mathrm{I}_{R 1}}{\mathrm{u}_{i n}}=\frac{L_{B} L_{m} s^{2}+\left(L_{B} R_{3}+L_{m} R_{5}\right) s+R_{2} R_{3}}{L_{B} L_{m} L_{1} s^{3}+c_{1} s^{2}+c_{2} s+R_{1} R_{2} R_{3}}
$$

where:

$$
\begin{aligned}
R_{4} & =\left(R_{1}+R_{2}\right), R_{5}=\left(R_{2}+R_{3}\right) \\
c_{1} & =\left(L_{m}\left(L_{B} R_{4}+L_{1} R_{5}\right)+L_{B} L_{1} R_{3}\right) \\
c_{2} & =\left(R_{2}\left(L_{m} R_{1}+L_{1} R_{3}\right)+R_{3} R_{4}\left(L_{B}+L_{m}\right)\right)
\end{aligned}
$$

\subsection{Mechanical Subsystem}

The mechanical subsystem is a typical second order system consisting of the moving mass of the element and any stiffness and damping within the system with an input force originating from the electrical subsystem. Using NEWTON's Law the mechanical subsystem can be described by the equation of motion given in equation (10).

$$
\ddot{\mathrm{x}}=\frac{1}{m} \mathrm{~F}-\frac{d}{m} \dot{\mathrm{x}}-\frac{r}{m} \mathrm{x}
$$

Using the current-force analogy, this mechanical subsystem can also be described by an equivalent circuit that has a current input analogous to the electrical force supplying three parallel components:

- a capacitance, $C_{m}$ representing the moving mass,

- a resistor, $R_{d}$ representing the damping within the mechanical system, as well as the damping caused by the velocity induced eddy currents,

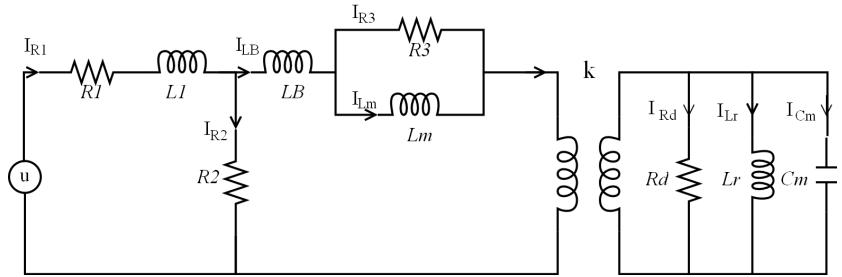

Figure 5. Final equivalent circuit.

- and an inductor, $L_{r}$ representing any stiffness within the system.

\subsection{Full Model}

The full model can be created by combining the two subsystems with the dynamics of the system. There are two equations that describe the flow of energy between the two subsystem circuits: the LORENTz force law and FARADAY's law of induction.

The current flowing perpendicular to the flux density results in a force known as the LORENTZ force:

$$
\mathrm{F}=B N l \mathrm{I}
$$

This force moves both the coil and the bobbin, therefore a force is generated by both $I_{1}$ and $I_{2}$ :

$$
\mathrm{F}=B N l \mathrm{I}_{1}+B N l \mathrm{I}_{2}=k\left(\mathrm{I}_{1}+\mathrm{I}_{2}\right)
$$

The magnetic flux density, $B$ is assumed to be constant over the travel of the coil/bobbin. The number of turns $N$ and the conductor turn length $l$ are also constant and so $\mathrm{BNl}$ may be combined to produce one force constant $k$. This force is the input to the mechanical subsystem.

As the coil and bobbin are allowed to move in the field, their movement will generate counter-electromotive forces within their circuits which can be expressed as below:

$$
\mathrm{E}=B N l \dot{\mathrm{x}}=k \dot{\mathrm{x}}
$$

The derivative $\dot{x}$ is the perpendicular component of the velocity of the wire relative to the flux lines. The voltage equations (6) and (7) are augmented as below to include the counter-electromotive force:

$$
\begin{aligned}
\mathrm{u}_{1} & =N_{1}\left(L_{M} \frac{d \mathrm{I}_{m}}{d t}+L_{1} \frac{d I_{1}}{d t}+L_{B} \frac{d}{d t}\left(\mathrm{I}_{1}+\mathrm{I}_{2}\right)\right)+R_{1} \mathrm{I}_{1}+\dot{\mathrm{x}} k \\
0 & =N_{2}\left(L_{M} \frac{d \mathrm{I}_{m}}{d t}+L_{B} \frac{d}{d t}\left(\mathrm{I}_{1}+\mathrm{I}_{2}\right)\right)+R_{2} \mathrm{I}_{2}+\dot{\mathrm{x}} k
\end{aligned}
$$

In the mechanical-electrical analogy, velocity is equivalent to voltage, and thus the voltage across the capacitor in the mechanical circuit is $\dot{x}$. As both the counter-electromotive force equation and the LORENTZ force equation have a factor of $k$, the transfer of force between the two subsystem circuits is equivalent to a transformer with a turns ratio of $\sqrt{k}$. The mechanical components can be transferred to the primary electrical side by multiplying them by the square of the turn ratio, producing the final equivalent circuit as shown in Figure 5. The final system parameters are displayed in Table 1. 
Table 1. System Parameters

\begin{tabular}{|c|c|}
\hline Symbol & Meaning \\
\hline \hline$R_{1}$ & Coil winding resistance \\
\hline$L_{1}$ & Coil inductance \\
\hline$R_{2}$ & Bobbin (eddy current) Resistance \\
\hline$R_{3}$ & Core loss resistance \\
\hline$L_{B}$ & Mutual inductance \\
\hline$L_{m}$ & Resistor equivalent of mechanical damping \\
\hline$R_{d}$ & Inductor equivalent of mechanical friction \\
\hline$L_{r}$ & Capacitor equivalent of moving mass \\
\hline$C_{m}$ & Force constant \\
\hline$k$ & \\
\hline
\end{tabular}

From the equivalent circuit, the following state-space expression can be formed:

$$
\left[\begin{array}{c}
\dot{\mathrm{I}}_{R 1} \\
\dot{\mathrm{I}}_{L B} \\
\dot{\mathrm{I}}_{L 3} \\
\ddot{\mathrm{x}} \\
\dot{\mathrm{x}}
\end{array}\right]=\left[\begin{array}{ccccc}
\frac{-R_{4}}{L_{1}} & \frac{R_{2}}{L_{1}} & 0 & 0 & 0 \\
\frac{R_{2}}{L_{B}} \frac{-R_{5}}{L_{B}} & \frac{R_{3}}{L_{B}} & \frac{-k}{L_{B}} & 0 \\
0 & \frac{R_{3}}{L_{m}} & \frac{-R_{3}}{L_{m}} & 0 & 0 \\
0 & \frac{k}{C_{m}} & 0 & \frac{-k}{C_{m} R_{d}} & \frac{-k}{C_{m} L_{r}} \\
0 & 0 & 0 & 1 & 0
\end{array}\right] \bullet\left[\begin{array}{c}
\mathrm{I}_{L 3} \\
\mathrm{I}_{L 3} \\
\dot{\mathrm{x}} \\
\mathrm{x}
\end{array}\right]+\left[\begin{array}{c}
1 \\
\frac{\mathrm{I}_{1}}{1} \\
0 \\
0 \\
0 \\
0
\end{array}\right] \bullet \mathrm{u}_{\mathrm{in}}
$$

\section{MODEL VERIFICATION \& PARAMETER IDENTIFICATION}

Frequency sweeps were made on a single actuation element in order to determine the parameter values and verify the model. Three signals were measured: the position and acceleration of the rod, and the coil current. Frequency responses for these signals were obtained over the frequency range $1-2000 \mathrm{~Hz}$ with the coil free-moving. A frequency sweep for coil current was also carried out with the coil clamped mid-way along its travel. This aids the identification process as clamping the coil removes the mechanical dynamics from the system.

Only two parameters could be measured directly: the moving mass, $C_{m}$ and the force constant, $k$. The moving mass was weighed and the force constant determined by applying a known current to the element and measuring its force using a scales. These two parameters determine the capacitance $C_{m}$ as $C_{m}=m k^{2}$.

The remaining parameters were found by fitting the model to the frequency response data using the optimisation toolbox. The frequency data was entered into Matlab and weights were applied to favour the magnitude response and the $10-100 \mathrm{~Hz}$ region and remove the influence of the high frequency regions in the position/acceleration responses. Known model values were set and the remaining parameters defined as values to be determined. The model response was then matched to the measured data by defining the difference between them as a scalar function, and using the Matlab function 'fsolve' to find a minimum of the function through variation of the parameters, starting from an initial estimate.

The clamped frequency response was used first in the fitting process, as this system has fewer parameters. The clamped system transfer function was stated in equation (9). The results suggested that the effect of the inductance $L_{B}$ (symbolising the flux linking the bobbin
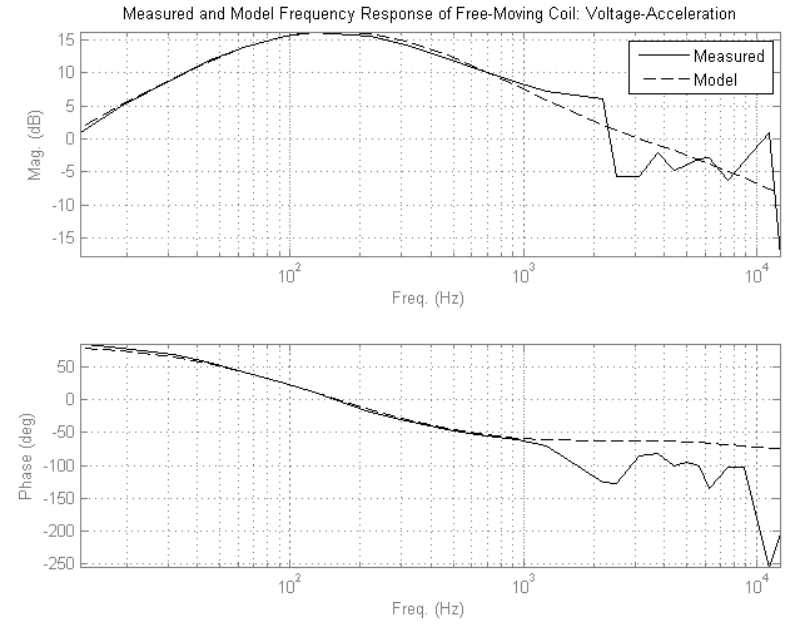

Figure 6. Model and Experimental Frequency Response of Free-Moving Coil: Voltage-Acceleration.
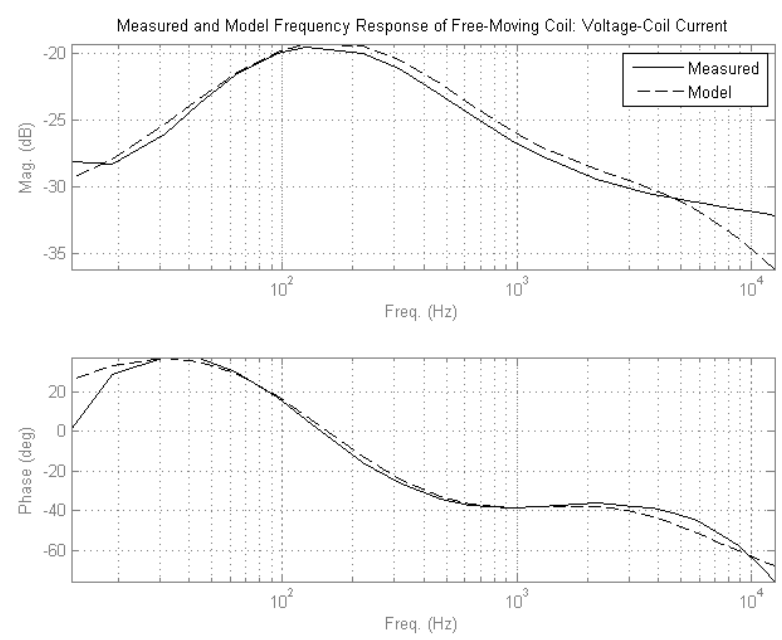

Figure 7. Model and Experimental Frequency Response of Free-Moving Coil: Voltage-Current.

and the coil) on the system was negligible. Thus, $L_{B}$ was removed from the model, simplifying it by a degree. The new clamped subsystem model is as described in equation (17). Hence, the parameters $L_{m}, L_{1}, R_{1}$ and $R_{2 \mid 3}$ were determined from this response.

$$
\frac{\mathrm{I}_{R 1}}{\mathrm{u}_{\text {in }}}=\frac{L_{m} s+R_{2 \mid 3}}{L_{m} L_{1} s^{2}+\left(L_{m}\left(R_{2 \mid 3}+R_{1}\right)+L_{1} R_{2 \mid 3}\right) s+R_{1} R_{2 \mid 3}}
$$

where:

$$
R_{2 \mid 3}=\frac{R_{2} R_{3}}{\left(R_{2}+R_{3}\right)}
$$

The free-moving current and position responses were used to determine the remaining model parameters. The ratio between $R_{2}$ and $R_{3}$ was determined, allowing values for each to be found. The mechanical parameters $L_{r}$ and $R_{d}$ did not have a significant affect on the system and thus are set very high. The frequency responses of the model and measured data for the free-moving system are shown in Figures 6 and 7.

The model provides a good fit to the measured data between $5-100 \mathrm{~Hz}$, which is the critical frequency range. 


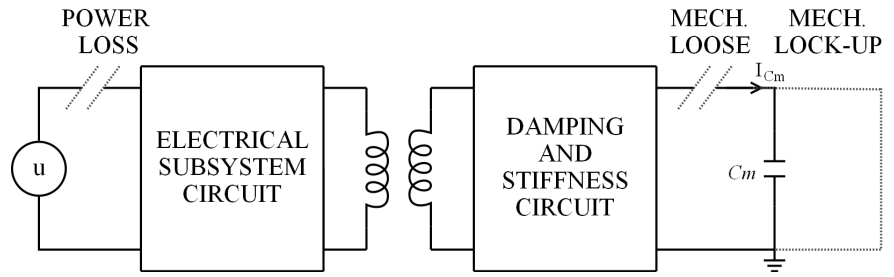

Figure 8. Faults represented in the equivalent circuit a single actuation elements.

The discrepancies present in the acceleration and position response at higher frequencies are due to unmodelled mechanical resonances. There may also be some skin effects present in the high frequencies, which could be modelled. However, this would increase the model order significantly.

In the acceleration frequency response, a discrepancy below $5 \mathrm{~Hz}$ can also be observed. This difference is attributable to stiction. Again, the inclusion of the stiction dynamics in the model is not considered worthwhile as this would introduce non-linearities into the system. However, transient response data suggests that the stiction is significant and that its inclusion in the model may be necessary in the future.

\section{FAULT MODELLING IN A SINGLE ELEMENT}

As the HRA is being developed in the interest of fault tolerance, it is necessary to model potential faults that can be injected into the system. Three main fault cases have been identified and modelled to date, namely:

- Mechanical Loose - A mechanically loose actuation element loses the ability to exert force between its two end points. Thus, a mechanically loose element behaves as if it is not there.

- Mechanical Lock-up - An element loses the ability to change the length between its two end points. This may occur if the coil of the first actuation element is deformed and touches the magnet. This fixes the mass with respect to the reference point, and consequently the relative position and the speed are constant.

- Power Loss - This fault is where the electrical input to the actuation element is lost, or the coil circuit becomes open circuit, but the mechanical subsystem continues to operate.

These faults are easily represented in the electrical equivalent circuit format. Figure 8 illustrates where the equivalent circuit for an actuation element needs to be shorted or severed to represent the given faults. A loss of power is realised by breaking the circuit so that the electrical power supply is disconnected. The mechanical loose fault is similar, as the force applied to the mass is lost. As current is equivalent to force in the current analogy, and the capacitance $C_{m}$ represents the mass, the current supply to this component $\mathrm{I}_{C m}$ must be removed, and thus the circuit is opened at this point. The mechanical lock-up fault requires the capacitor to be short circuited: the force applied to the mass is bypassed, fixing the masses velocity and position relative to the preceding element or surface.

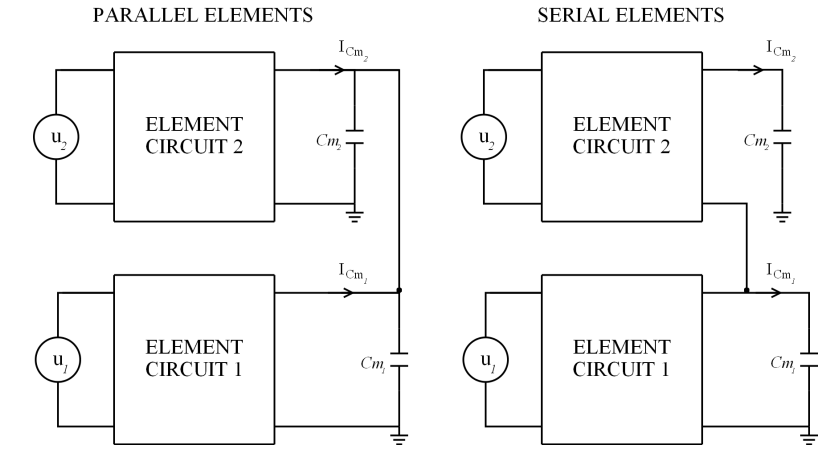

Figure 9. Parallel and serial assemblies using circuit analogies.

\section{MODELLING OF ACTUATION ASSEMBLIES}

Having developed a model for a single actuation element, multi-element assemblies can now be constructed to form a HRA. The current focus of the project is on planar assemblies and thus the elements are arranged either serially, or in parallel, or in serial/parallel combinations. The optimum configuration of actuation elements, in accordance with the high redundancy actuation concept, is discussed in [Steffen et al., 2007a], and hence will not be addressed here. This section will merely address the issue of creating models of possible actuation assemblies using SMAC moving coil actuators as actuation elements.

The use of electrical analogies in the model derivation allows multi-element actuator models to be created by replicating and interconnecting the equivalent circuit according to the assembly structure. For example, if two actuation elements are arranged in parallel and act upon a common load, their forces add and act upon one moving mass. Thus, the element equivalent circuit capacitor branch currents $\mathrm{I}_{C m 1}$ and $\mathrm{I}_{C m 2}$ add and flow through one combined capacitor, or alternatively each current flows separately through two parallel capacitors that add to make the moving mass as shown in Figure 9.

If actuators are connected in series, the first moving mass has the force of the first element and an opposing force from the second element acting upon it, and the second moving mass has the second element force applied to it. In electrical equivalence terms this means the first capacitor $C_{m 1}$ has the current $\mathrm{I}_{C m 1}-\mathrm{I}_{C m 2}$ and the second capacitor $C_{m 2}$ has the current $\mathrm{I}_{C m 2}$ and thus the two circuits are connected as shown in Figure 9.

More complicated assemblies can be modelled based on these two fundamental circuits.

\section{FAULTS IN ACTUATION ASSEMBLIES}

The equivalent circuit representation of the model provides an intuitive insight into the effect of the faults on multielement assemblies. To illustrate this point, the equivalent circuit fault model for a two-by-two series-in-parallel system, as shown in Figure 10, is given in Figure 11.

In the example assembly, elements one and two work upon masses $m_{1}$ and $m_{2}$ respectively. $m_{1}$ and $m_{2}$ are the combined mass of the moving mass of elements 1 and 2 the casing mass of 3 and 4, respectively. The casing masses of 


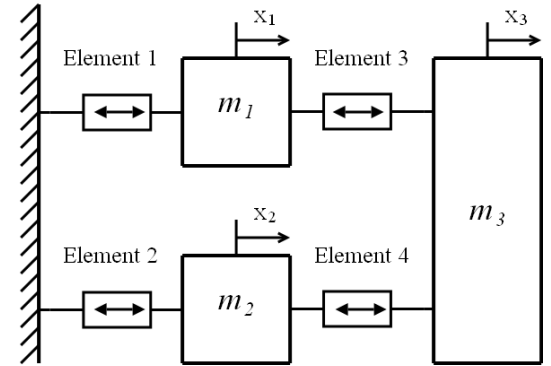

Figure 10. Two-by-two series-in-parallel assembly.

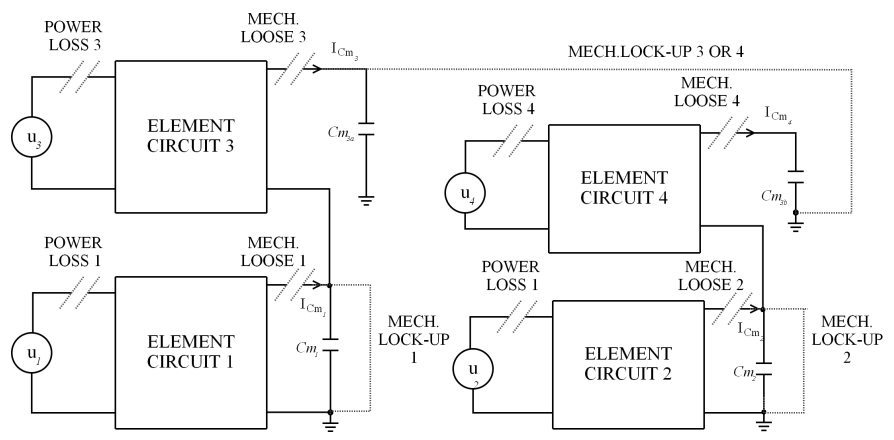

Figure 11. Faults represented in the equivalent circuit for a two-by-two series-in-parallel assembly.

actuation elements 1 and 2 are not included in the diagram as they are fixed to a surface. actuation elements 3 and 4 both apply their force to $m_{3}$, which is the combined mass of the moving masses of elements 3 and 4 and the load mass. The effect of faults on this system, with reference to the equivalent circuit diagram, is discussed below.

\subsection{Power Loss}

A power loss fault in any of the actuation elements removes the influence of the electrical subsystem on the associated mechanical subsystem, but does not affect the flow of force between the systems. Hence theoretically, this HRA can withstand up to three power losses without complete loss of force to the load.

\subsection{Mechanical Loose}

A mechanical loose fault in actuation elements 1 or 2 results in loss of current to $C_{m 1}$ or $C_{m 2}$ respectively. In mechanical terms, this means that the force on $m_{1}$ or $m_{2}$ is lost, rendering that serial branch inoperable. The same applies to elements 3 and 4 , as a loose fault in either will result in a reduction of current to $C_{m 3(a+b)}$. However, the remaining un-loose serial branch will continue to provide force to the load mass in either case, resulting in a theoretically operational system.

If both serial branches suffer a loose fault however, current to $C_{m 3(a+b)}$ will be lost completely, resulting in the failure of this HRA configuration.

\subsection{Mechanical Lock-up}

A mechanical lock-up in elements 3 or 4 results in the short-circuiting of the capacitance $C_{m 3(a+b)}$, hence fixing $x_{3}$ and $\dot{x}_{3}$ with respect to the previous masses. If elements
1 or 2 lock-up, then their respective capacitor is shortcircuited and thus the states of the associated mass are fixed with respect to the fixed surface. The system will remain theoretically operational as long as one element in each serial branch remains un-locked.

These observations confirm the logical deductions that parallel elements reduce the effect of loose mechanical faults, but do not aid fault tolerance in the case of mechanical lock-ups. Conversely, serial elements improve fault tolerance to mechanical lock-ups, as the other element remains effective, but are vulnerable mechanically loose faults. The quantification of fault tolerance within high redundancy actuators is further discussed in [Steffen et al., 2007b].

\section{CONCLUSIONS}

In this paper a model for a moving coil actuator has been derived with the intention of using it as a single element within an electro-magnetic HRA. The model was verified using experimental data and the parameter values were identified. The modelling of faults appropriate to moving coil technology in a single element was considered and the formulation of assembly models using equivalent circuits was discussed. Finally, the effect of faults on multi-element assemblies was considered and an example given. This modelling provides a foundation for the control studies planned for the future. The control studies planned within the project take two directions: robust control strategies and self-organising control.

\section{ACKNOWLEDGEMENTS}

This project is a cooperation of the Control Systems group at Loughborough University, the Systems Engineering and Innovation Centre (SEIC), and the actuator supply SMAC UK Ltd.. The project is funded by the UK's Engineering and Physical Sciences Research Council (EPSRC) under reference EP/D078350/1.

\section{REFERENCES}

M. Blanke, M. Staroswiecki, and N. E. Wu. Concepts and methods in fault-tolerant control. American Control Conference, 2001.Proceedings of the 2001, 4, 2001.

H. D. Chai. Electromechanical motion devices. Prentice Hall, United States, 1998.

X. Du, R. Dixon, R. M. Goodall, and A. C. Zolotas. Lqg control of a highly redundant actuator. In Conference for Advanced Intelligent Mecatronics, 2007.

R. J. Patton. Fault detection and diagnosis in aerospace systems using analytical redundancy. Computing and Control Engineering Journal, 2(3), 1991.

SMAC. Actuator data sheet - electric linear/rotary moving coil actuators lal90-50, 2004.

T. Steffen, J. Davies, R. Dixon, R. M. Goodall, and A. C. Zolotas. Using a series of moving coils as high redundancy actuator. In IEEE Conference for Advanced Intelligent Mechatronics, Zurich, 2007a.

T. Steffen, R. Dixon, R. M. Goodall, and A. C. Zolotas. Quantifying the fault tolerance of high redundancy actuator assembly. Submitted to Mechatronics, 2007b. 